\title{
Los conflictos y la vida diaria
}

Conflicts and everyday life

Os conflitos e a cotidianidade

DOI: https://doi.org/10.21803/pensam.v12i22.254

Alfredo Islas Colín https://orcid.org/0000-0002-2841-4315

Oscar Pérez Baxin https://orcid.org/0000-0002-4332-2427

\section{¿Cómo citar este artículo?}

Islas, A. \& Pérez, O. (2019). Los conflictos y la vida diaria. Pensamiento Americano, 12(23) 169-182. DOI: https://doi.org/10.21803/pensam.v12i22.254

\section{Resumen}

La cotidianidad de las personas en el mundo actual, ha llevado a la creación de nuevos esquemas para actuar y construir reglas que permitan sobrevivir a lo complejo de la globalización, los conflictos que se generan en el transitar en diversos escenarios tienen posturas y percepciones variadas, lo cual plantea a las personas una toma de decisiones que resultan nocivas para la sana convivencia y el de crear una cultura de paz. Las reformas constitucionales de 2008 en México, en materia de justicia alternativa han planteado que los justiciables cuenten con métodos autocompositivos para solucionar los conflictos.

En tal sentido el presente artículo realiza una revisión teórica de los conflictos, desde la disciplina de los mecanismos alternativos de solución de controversias, exploramos cómo los conflictos están presentes en nuestra cotidianidad y son más próximos en el mundo globalizado. Este artículo es resultado del proyecto: Los mecanismos alternativos de solución de controversias en Tabasco, desarrollado por el Grupo de Investigación de mecanismos alternativos de solución de conflictos y derechos humanos.

Palabras Clave: Mediación, conflictos, cotidianidad, negociación, violencia.

\section{Abstract}

The daily life of people in today's world, has led to the creation of new schemes to act and build rules that allow the globalization complex to survive, the conflicts that are generated in transit in various scenarios have varied positions and perceptions, which raises people to make decisions that are harmful to healthy living and to create a culture of peace. The constitutional reforms of 2008 in Mexico, in the field of alternative justice, have proposed that the justiciable have self-compliant methods to solve the conflicts.

In this sense, this article makes a theoretical review of conflicts, from the discipline of alternative dispute resolution mechanisms, we explore how conflicts are present in our daily lives and are closer in the globalized world. This article is the result of the project: Alternative dispute resolution mechanisms in Tabasco, developed by the Research Group on alternative dispute resolution and human rights mechanisms.

Key words: Mediation, conflicts, everyday life, negotiation, violence. 


\section{Resumo}

A vida cotidiana das pessoas no mundo de hoje levou à criação de novos esquemas para atuar e construir regras que permitem que o complexo da globalização sobreviva. Os conflitos que são gerados no trânsito de diversos cenários têm posições e percepções variadas, e leva as pessoas a tomarem decisões que são prejudiciais à vida saudável e para a criação de uma cultura de paz. As reformas constitucionais de 2008 no México, no campo da justiça alternativa, propuseram que os justiçáveis têm métodos autocomplacentes para resolver conflitos.

Nesse sentido, este artigo faz uma revisão teórica dos conflitos, a partir da disciplina de mecanismos alternativos de resolução de controvérsias, exploramos como os conflitos estão presentes em nosso cotidiano e são mais próximos no mundo globalizado. Este artigo é o resultado do projeto: Os mecanismos alternativos de resolução de conflitos em Tabasco, desenvolvido pelo Grupo de Pesquisa sobre mecanismos alternativos de resolução de conflitos e direitos humanos.

Palavras-chave: Mediação, conflitos, cotidianidade, negociação, violência

\section{Perfil}

Licenciado en Derecho. Magister en Derecho constitucional por la Université Panthéon-Assas, París II. Maestría en Derecho Constitucional por la Universidad de Castilla-La Mancha. Investigador Nacional, nivel III (SNI-CONACYT). Profesor Investigador de la Universidad Juárez Autónoma de Tabasco (UJAT)

\section{Perfil}

Licenciado en Derecho. Profesor investigador perfil prodep de la UJAT. Doctor en Métodos de Solución de Conflictos y Derechos Humanos. opbaxin@ gmail.com

\section{Alfredo Islas Colín}

Ph.D en Derecho constitucional

\section{Oscar Pérez Baxin}

Ph.D en Métodos de Solución de Conflictos y Derechos Humanos. 


\section{Introducción}

El presente trabajo trata de acercarnos al conocimiento del conflicto desde la postura de los mecanismos alternativos de solución de controversias, tal como aparece plasmado en el artículo 17, párrafo cuarto de la Constitución Política de los Estados Unidos Mexicanos, de la manera siguiente: Las leyes preverán mecanismos alternativos de solución de controversias. En la materia penal regularan su aplicación, aseguraran la reparación del daño y establecerán los casos en los que se requerirá supervisión judicial.

A partir de la norma constitucional citada, hemos dividido en tres partes la exposición, 1.- el acercamiento con la vida diaria, pero considerando aspectos teóricos de diversos autores, de ahí partimos para buscar algunas coincidencias, que nos acerquen a la comprensión del mismo y lograr el objetivo del artículo de abrir la discusión en torno a los mecanismos alternativsos de solución de controversias. 2.Aquí vemos a los conflictos en la cotidianidad, como parte de una era global, que nos ha llevado a acercarnos a todos pero también a ser más encerrados en nosotros mismos, y creando nuevos sujetos sociales. Al final el punto 3.- Se refiere a como vemos el conflicto en la actualidad.

\section{Metodología}

El Método utilizado en el presente artículo fue el interpretativo, que consiste en reconocer a partir de elementos teóricos y epistemológicos que proporcionan elementos válidos a los mecanismos alternativos de solución de controversias, el conflicto y la cotidianidad.

Los resultados presentados pretenden aportar interpretaciones desde la perspectiva de los autores y las discusiones generadas en torno al grupo de investigación sobre derechos hu- manos, mecanismos alternativos de solución de controversias y justicia restaurativa.

\section{Resultados}

\section{1.- Cotidianidad del conflicto}

A lo largo de la historia cada sociedad ha tenido una suerte de confrontaciones, de lograr acuerdos según las difirencias en muchos sentidos, la pugna de intereses y la creación de polémicas, de disputas se debe a intereses de hacer que una idea se la que prevalezca por otras (Alzate, 1998). Debido a estas situaciones en consenso cada sociedad se ha dedicado a la elaboración de sistemas que tengan como fin el de realizar de regulaciones para la convivencia pacífica y que debido a ello no se realice elementos de la cohesión social para que se transformen los conflictos que tienden dañar a la vida colectiva y para quienes se encuentren involucrados, cambien las ideas sobre los mismos y les brinden un sentido positivo de ver los conflictos, no pensar que son negativos en sentido general.

En tal sentido el conflicto debe ser una oportunidad para realizar cambios significativos, que tiendan a ver el progreso y en tal sentido la mediación como proceso para la gestión participada y pacífica del conflicto, puede ser una condición constitutiva de las relaciones sociales (Martínez, 1999). Por su parte Cornelio (2017), refiere que el conflicto o los conflictos se manifiestan como un fenómeno complejo pero que nos muestra un medio de aprendizaje en la acción o hecho del conflicto.

La reflexión del ejercicio del conflicto pone en evidencia que la relación existente entre las partes al igual que el significado que cada una de ellas aporta, proporciona a la controversia, dentro del orden pragmático los medios alternos a la solución del conflicto desencadenan un orden legitimo otorgado a la 
ciudadanía para que sean ellos mismos quienes solucionen sus problemas.

La psicología social comunitaria al hablar de conflicto lo refiere en Wright (2012) como la percepción de incompatibilidad entre el objetivo que una de las partes en el conflicto pretende y en contraparte el objetivo de tal modo que cada una de ellas mantiene la idea de que la pretensión de la otra parte le resulta risgosa para sus propios intereses.

Así tenemos que para que haya un conflicto no se precisa la presencia de una relación previa entre las partes, es decir que solo basta un vínculo que se establece entre ellas cuando una de ellas considera que las pretensiones de la otra son perjudiciales para sus intereses.

Pero debemos de tomar en cuenta que los conflictos también son frecuentes entre personas que mantienen diversos tipos de relaciones y, por tanto se da entre ellas una interdependencia (Hoyo, 2006).

Con ello damos por hecho que en el proceso de la resolución constructiva de los conflictos se ejecutan maniobras, que son reflexiones cognoscitivas (Ury, 1996) y que en ellas se haIlan las siguientes:

1. La estrategia ganar-ganar. Las partes observan una actitud de total cooperación. En apariencia, esta es una situación muy difícil de lograr.

2. La estrategia ganar-perder. Cada parte, con su enfoque, intentará resolver el conflicto a su favor, sacara provecho de la situación y salir beneficiada. Es evidente que aquí una parte saldrá perjudicada.

3. La estrategia perder-perder. Es en donde ninguna de las dos partes obtiene su objetivo. Las partes piensan: "la negociación se da como yo desee", y no hay resultado alguno. Ambas partes consideran evitar un conflicto es preferible a confrontar directamente el problema.

El conflicto según Baruch y Folger (1996), suministra a las personas una oportunidad para desarrollar y ejercitar la autodeterminación y la confianza en las propias fortalezas, además de que proporciona a las personas una ocasión para desarrollar y mostrar respeto y consideración mutuos, y además de que contiene valiosas oportunidades en ambas dimensiones del crecimiento moral, quizás en grado más alto que en la mayoría de las restantes experiencias y relaciones humanas.

En el terreno de la mediación, según Lederach (1984) se ha llegado además a diferenciar entre gestión, resolución y transformación del conflicto y resume así el significado de cada término:

Sobre el término de resolución de conflictos, el aporte del autor estriba en que el concepto en sí indica la necesidad de entender como un todo el desarrollo del conflicto es decir cuando empieza y cuando termina el mismo, en tal sentido lo que se busca es una convergencia de los intereses de cada uno de los actores, entendiendo que al afrontar el conflicto y tal como lo refiere el de erradicar el mismo; claro que da una idea más de que en algunos casos no todo puede llegar a resolverse, y además señala que tampoco el camino para llegar a esa resolución es en general tan despejado, ya que éste genera su propia dinámica, pues en la búsqueda de las soluciones hay que atravesar por crisis acompañadas de diversas emociones que no permiten llegar directamente a decisiones definitivas.

Con respecto a la gestión de los conflictos, el autor reconoce que el conflicto no puede resolverse en el sentido de librarse de él, no 
debemos verlo en ese sentido, y el mismo autor pone el acento en limitar las consecuencias destructivas del conflicto, cuando es visto desde un sentido. $Y$ también hace referencia a una aproximación de tipo práctico y de control, por la cual se intenta contener o mantener el conflicto dentro de límites aceptables, intentando realinear las divergencias, dentro de su forma de abordar el conflicto el mismo sabe de las situaciones liminales que existen y que debemos de considerar.

Con respecto a la transformación de conflictos, el autor pone el acento en la naturaleza dialéctica del conflicto, reconociéndolo como un fenómeno que forma parte natural de las relaciones humanas, y que dentro de las relaciones interpersonales es donde lo podemos encontrar con todas sus manifestaciones. Es decir que para el autor el conflicto es un elemento necesario en la construcción y reconstrucción de las realidades de los sujetos sociales, y cuyos patrones destructivos, visto desde un primer plano, pueden ser canalizados hacia una expresión constructiva.

La transformación tal y como la plantea Puntes (2007), es un concepto descriptivo de la dinámica del conflicto, y la refiere como una máquina y oportunidad de transformación, pero al mismo tiempo en que es prescriptivo por los propósitos que persigue la construcción de soluciones; sugiere una comprensión dinámica del conflicto, en el sentido de que puede moverse en direcciones constructivas o destructivas, de ahí la importancia del conocimiento del especialista en la materia, por lo que debera reconocer que, con frecuencia, el conflicto no va a desaparecer ni a ser completamente resuelto, la parte ideal es que éste sea resuelto, pero hayq ue considerar que el proceso de solución no está sujeto a un tiempo específico y que además no puede ser controlado como quisieran las personas involucradas o los grupos participantes; por el contrario, el estar inmersos en una relación, el conflicto es un proceso vivo e imprevisible, y se va a ir moviendo y transformando según se avance con una buena negociación. En otro sentido el conflicto puede ser transformado en algo antes impensado y se convierte entonces en una parte de la vida y no en asunto que debe ser eliminado por ser destructivo e indeseable, ya que esta es una posición reduccionista del mismo, esto considerado por Redorta.

Con estas dos consideraciones, la propuesta de Puntes (2007) y de Redorta (2006), se está de acuerdo en que tanto los conflictos como las soluciones son una construcción de los participantes, en donde la ayuda del especialista se verá reflejada, ellos los involucrados al ser mediados podrán realizar los cambios, mediante un aprendizaje al saber que el conflicto nos lleve a evolucionar. En tal sentido actitud y habilidades personales y grupales, ayudan a que se asuman responsabilidades y en este sentido las decisiones con respecto al procesamiento de las diferencias en su vida y para usar en consecuencia métodos que favorezcan la transformación de los conflictos y que las personas involucradas los puedan entender.

Por lo tanto tenemos que el objetivo principal de la resolución, la gestión y la transformación, está triada es la de producir cambios en las actitudes de las partes implicadas para que el conflicto sea reconocido con sus aspectos positivos y negativos, de manera que una vez reconocido, éste sea trabajado para crear una agenda amplia con los temas y subtemas que aporten elementos que nos permitan el reconocimiento de los intereses y pasar a crear acuerdos desde las posturas de los involucrados lo cual les llevará a comprometerse en cada parte de lo acordado por éstos. 


\section{2.- Conflictos en el mundo globalizado}

Hablar de conflicto es un tema actual que podemos encontrar en todos lados, en las conversaciones cotidianas y a donde quiera que vayamos, pero los conflictos siempre han existido en la historia de la humanidad, podemos mencionar al igual que muchos autores refieren que éste es inherente al género humano, tanto de manera interna como externa, y que está presente desde nuestro nacimiento, es decir que: el conflicto nace con el propio hombre, pues al nacer ya tenemos el primer conflicto al abandonar el seno materno que tan cómodamente nos aloja y al reconocer un medio hostil se entra en conflicto (Gottheil \& Schiffrin, 1996).

Los conflictos también se pueden interpretar como procesos sociales que reflejan la forma en que se relacionan las personas o grupos sociales. Entre las causas más frecuentes de conflictos están según Vinyamata los siguientes:

1. El acceso a la distribución de recursos (territorio, dinero, fuentes de energía, alimentos $\mathrm{u}$ otros).

2. El control del poder y la participación en la toma de decisiones políticas (en organizaciones, en partidos o en cualquier otro grupo organizado).

3. La defensa de la identidad (cultural, política, religiosa o de otra índole).

4. La defensa del estatus o posición social (en espacios como los sistemas de gobierno, las jerarquías religiosas, las organizaciones políticas) (Vinyamata, 2005).

El conflicto corresponde propiamente al género humano, pero los tiempos actuales le han proporcionado una nueva dimensión cualitativa, ya que la cultura como se ha venido moviendo en los sentidos tecnológicos, de mercado y de las comunicaciones le aporta nuevas vías para la gestión de algunas de las problemáticas con las que se enfrentan los nuevos sujetos sociales, siendo, a su vez, una respuesta "conflictiva" porque se interacciona directamente con todo lo existente previamente. De esta manera la cultura humana es por definición conflictiva (Redorta, 2004). Con la transmisión de las normas culturales, las propuestas "naturales" se ven condicionadas y modificadas. Evidentemente las situaciones son continuamente "nuevas" por los cambios habidos en el medio y por los "avances" generados en la propia historia de la humanidad, en la que se han ido modificando las formas de organización social, las normas de conducta y la tecnología, como lo referiamos líneas arriba, prro además como los humanos hemos poblado prácticamente todo el planeta, se han producido variaciones de acuerdo con los distintos ambientes, situaciones y evoluciones locales, creando nuevos sujetos sociales (Rodríguez, 2017).

El conflicto es una constante de la vida social en la que se comparten necesidades y expectativas., así como perccepciones y formas de interpretar el día a día. Las formas en que se manifiestan los conflictos a lo largo del tiempo y alrededor del mundo en la actualidad son muy variadas. Se transmiten de padres a hijos, de generación en generación, se crean dentro de cada cultura, de cada sociedad, como experiencias y aprendizajes exitosos de gestión de las condiciones materiales y sociales de vida, es verdad que la socialización provee de muchos elementos para la vida social y que lo hagamos de la menor manera, pero también aportan aquellos elementos intangibles de generación de conductas conflictivas.

Los humanos no sólo participan de la conflictividad sino que se convierten en agentes

Pensamiento Americano Vol. 12 - No. 23 • 2019 • Enero - Junio • Corporación Universitaria Americana • Barranquilla, Colombia • ISSN: 2027-2448 · 
de regulación, transformación y creación de conflictos. En tal sentido Ross (1995) señala que el conflicto tiene que ver con los fines concretos que los adversarios persiguen $\mathrm{y}$, al mismo tiempo, con las interpretaciones que éstos hacen de lo que está en disputa. La mayoría de las veces el asunto en controversia es el punto focal de soterradas diferencias de las que los antagonistas sólo tienen un conocimiento parcial; diferencias éstas que, si se ignoran, es muy probable que más adelante salgan de nuevo a la superficie, y así se hace, no por el hecho de querer crear conflictos sino que los vemos como parte de un todo.

En consecuencia, la especie humana puede ser definida como "conflictiva" en la medida en que tales contraposiciones están siempre presentes en todas las sociedades y actividades humanas es más, cabe proyectar su presencia a todo el tiempo y espacio humano (Redorta, 2006), desde que la "cultura" nos hizo humanos, nos ayudó a avanzar en el dominio de la naturaleza. El conflicto humano es más complejo y hace, igualmente, más complejo a todo su entorno, lo importante de ello es buscar las raíces de éstos y que se les pueda dar un tratamiento como algo positivo que nos mostrará las fortalerzas y debilidades de cada uno de los implicados,

Mientras que la noción de conflicto es clave y eje central de la propuesta terminológica de la psicología social. Cuando se habla de éste en dicha materia es porque se refiere a un amplio espectro que en la interacción e interrelación humana va desde situaciones y cuestiones que se elaboran con cierta rapidez, casi sin "problema" (aun cuando toda satisfacción de necesidades es búsqueda, mediatez, y por lo tanto es problema), hasta situaciones complejas que derivan en complicaciones, antagonismos, llegando a veces a estereotipias, rigideces o cristalizaciones, cuya posibilidad de resolución requiere la consulta o intervención de operadores (terceros) que aporten elementos o instrumentos eficaces para la superación del conflicto, tal y como se realizan en la utilización de mecanismos alternativos de solución de controversias.

E. De Bono (2012) considera que el problema del conflicto no se encuentra en el conflicto mismo, sino en las creencias de las personas, dice al respecto sobre las creencias que éstas son muy difíciles de erradicar o alterar en las personas, y sucede porque a menudo las personas optan por mantener una creencia y no aceptar la evidencia de lo que ha sido visto yu procesado por sus sentidos, son creencias y percepciones arraigadas en los grupos sociales y que impactan el carácter y actuación de las personas. Es así que el conflicto se manifiesta cuando un sistema de creencias cree que los valores que de él se desprenden deben ser aplicados en todas partes y adopta como misión hacer que esto suceda en la realidad inmediata, actuando de manera natural y en tal sentido la comprensión del conflicto es más compleja.

Lo más interesante de este autor es su propuesta de dos neologismos, y que corresponden a la de-conflicción que sería entendida como la disipación o eliminación de los fundamentos de los conflictos. Y la conflicción la cual correspondería a establecer, estimular y promover el conflicto, es decir conocer las raíces del mismo y acercarse al mismo con las implicaciones que ello acarrearía, es decir la comprensión y el manejo adecuado del conflicto.

En un sentido más restringido la noción de conflicto podríamos decir que conflicto es un choque de creencias o valores o intereses o direcciones. En este caso la conflicción estaría ligada a todo aquello que precede a ese choque pero no en una "espontaneidad" del mismo sino a un proceso deliberado en el que se 
"construye" el conflicto. La de-confliccion sería la tarea de desarticulación de los elementos precedentes (fundamentos incluidos) que generan el choque para derivarlos, ya no a una negociación o acuerdo o resolución, sino a un proyecto que trasciende hacia una alternativa o perspectiva nueva y diferente. De Bono (2012) precisamente, habla de proyectos como la mejor manera de resolver conflictos.

Según Sara Cobb (1995), los conflictos en general deben ser entendidos como un fenómeno discursivo, es decir como un proceso en donde se involucran por un lado la interacción de historias, la cual acaba por ser rotulada como problema por el sistema en el interior del cual estas historias son contadas, pero agrega que los conflictos son definidos como tal por el sistema en cuyo interior son observados, es decir las narrativas son importantes en la forma en que comprendemos el conflicto de acuerdo a dicha autora. Los profesionales de la práctica de resolución de los conflictos que ellos median; están más bien activa y paradójicamente involucrados en la construcción del conflicto como tal, así que cada conflicto posee una historia personal construida por las personas, de las cuales Suáres (1996) refiere que es una incompatibilidad entre conductas, percepciones, objetivos y/o afectos.

Pero para la mediación lo que interesa es la incidencia, prevalencia o presencia del conflicto en las personas y su cotidianidad, nuestra cultura en el nuevo milenio posee una tendencia a identificar el conflicto con sentimientos de hostilidad, animadversión, antipatía, odio y también con manifestaciones de violencia; sentimientos que en su mayoría suelen tener para nosotros, connotaciones claramente negativas, pero además nos sirve como un mecanismo para la defensa en el día con día.

Existe la percepción casi automática condi- cionada por nuestra cultura y por el sentido de conflicto que tiene dentro de ella, refiere Puntes (2007) y Martínez (1999) que el conflicto, es la situación que sucede entre al menos dos partes interdependientes y que se caracteriza por unas diferencias percibidas sobre la misma realidad y que las dos partes evalúan como negativas.

Así los conflictos los encontramos en las personas en su día a día, dentro de sus relaciones con otras personas; en nuestra casa, en el vecindario, colonia o calle, en las instituciones donde convivimos y laboramos y se manifiestan en la vida de la persona alterando situaciones sociales de convivencia (Arboleda, 2017).

Los conflictos entre individuos, grupos y naciones son también inherentes a la naturaleza humana como afirmamos líneas arriba, siempre que hay interdependencia la raíz de un conflicto está presente. El conflicto como proceso social puede manifestarse de diversas maneras. Las problemáticas pueden abarcar desde situaciones interpersonales hasta confrontaciones internacionales. El conflicto (Gottheil \& Schiffrin, 1996) que es manejado de forma inteligente puede producir resultados valiosos, así como el que es mal manejado puede ser destructivo, y perjudicial pero si no se logra resolver y queda en estado latente éste podrá crecer y ser muy perjudicial ya que la raíz se encontraría imbricada en el pasado y para llegar a él, se necesitaría demasiado tiempo para conocerla.

A continuación veamos la diversidad de conceptos y definiciones acerca del conflicto, cabe mencionar al respecto, que no hay una definición que englobe todo lo que pudiera representar, veamos:

El conflicto es inherente a los humanos y a las relaciones que establecemos; el conflicto es dinámico y evoluciona, para ejemplificar esta

Pensamiento Americano Vol. 12 - No. 23 · 2019 • Enero - Junio • Corporación Universitaria Americana · Barranquilla, Colombia • ISSN: 2027-2448 · 
afirmación tenemos que: si nos referimos al problema con los vecinos de arriba, a las peleas con la hija adolescente, a las tensiones con sus suegros, a las disputas entre jóvenes en el bachillerato, a las tensiones y confrontaciones entre naciones, pero también en las relaciones de tránsito a nuestros lugares de trabajo, a casa o por esparcimiento.

Para Christoper Moore (1996), el conflicto es entendido como: Todos nos enfrentamos a situaciones diarias que, de un modo u otro, nos presentan cierto grado de conflictividad. De todas formas, la mayoría de ellas acaban resolviéndose de forma informal; los desacuerdos y problemas que se originan en casi todas las relaciones o bien se resuelven más o menos a satisfacción de las personas comprometidas, o bien se abandonan por falta de interés o falta de capacidad para solucionarlas.

La definición de conflicto según la Real Academia Española (2001) refiere que, conflicto es combate, lucha, pelea, enfrentamiento armado, problema, cuestión, materia de discusión. Apuro, situación desgraciada de difícil salida. Coexistencia de tendencias contradictorias en el individuo capaces de generar angustia, trastornos neuróticos.

Esta definición tiene componentes que no permiten la posibilidad de solución, es decir vives o mueres, y mantiene además una posición evolucionista. Para el mismo Salvador Puntes (2007), buscando conectar los elementos de la mediación propone que, el conflicto en términos humanos es una máquina de evolución que nos permite progresar, aprender y crecer. Lo cual es un avance significativo para la convivencia de un mundo global y con tendencias a la autodestrucción de los entornos sociales y ambientales.
De manera que en la actualidad se tiene una visión positiva acerca del conflicto entre los autores que empezaron su análisis tenemos a: Baruch y Folger (1996), los cuales refieren la complejidad y amplitud del concepto y que sienta las bases de algo llamado conflictología. ${ }^{1}$

Para Ross (1995), el conflicto es "como las acciones de dos o más partes que contienden por el control de materiales escasos o recursos simbólicos".

En relación a este contexto y con el mismo Ross (1995), se considera que el conflicto se produce, sin duda alguna, cuando hay una competencia por recursos materiales, aumentos de salario, el uso del agua, el control de la tierra; o en un conflicto familiar, por una herencia o la custodia de un hijo, igualmente, puede producirse con motivo de la competencia por recursos simbólicos: prestigio, honor, jerarquía. Hay conflicto cuando las partes involucradas perciben que tienen razones suficientes para disputar lo que sea, cuando se sientes justificadas por cualquier motivo para hacerlo, y entonces se posicionan para ganar a toda costa de aquello que consideran es su verdad.

Desde la óptica del litigio (Hernández, 2007) el conflicto se concibe como la divergencia percibida de intereses o creencias (sentimientos, pensamientos, acciones) que impiden a las partes alcanzar simultáneamente sus aspiraciones, amén de que el conflicto es la oportunidad para crecer y expandir nuestra consciencia.

De esta forma, la cultura, el orden social y las expectativas que las personas de una misma sociedad comparten son algunos de los factores que es necesario considerar en 1 Ciencia disciplinaria y transversal que compendia conocimientos científicos racionales e irracionales sobre los conflictos, sus posibles causas y maneras de facilitar su solución
pacífica y no violenta. Nace durante los años 70 del s. XX en los EE.UU. a raíz de la Guerra Fría.

Pensamiento Americano Vol. 12 - No. 23 • 2019 • Enero - Junio · Corporación Universitaria Americana • Barranquilla, Colombia • ISSN: 2027-2448 • $\mathrm{http}$ //publicaciones.americana.edu.co/index.php/pensamientoamericano/index 
una explicación del conflicto: en ellos es posible advertir de qué modo se enlazan los objetos de disputa con los motivos para disputar (Vélez, 2017).

El conflicto existe en tanto que estamos vivos y nos relacionamos. No hay una definición absoluta del conflicto, así como tampoco una clasificación definitiva de éste. El conflicto contiene elementos positivos y negativos, y será justamente por decidir tratarlo desde su visión positiva que lo veremos como una oportunidad de cambio y de crecimiento más que un problema a resolver, a ocultar o a evitar (Navarro y Navarro, 2016). Además son aspectos necesarios para la convivencia en el sentido que nos permite conocer los límites de los otros, de tal forma que citando a Juárez podemos decir que si no queremos vivir en los conflictos y para los conflictos, la máxima del Benemérito queda a tono. El respeto al derecho ajeno es la Paz.

Según Neil Katz (1993), el conflicto aparece cuando hay al menos dos partes implicadas, interdependientes, que experimentan fuertes emociones, que perciben los resultados de una situación dada como incompatibles, y que al menos una de las partes reconoce la incompatibilidad y la entiende como problemática.

Para Katz (1993), frente a un conflicto sólo hay alternativas basadas en las posiciones, por lo tanto es un sistema excluyente: la solución del conflicto significa que la alternativa que "gana" únicamente responderá a lo que pedía una de las partes. Éste es el llamado conflicto cero por algunos autores, por lo cual la ganancia de uno es exactamente la pérdida del otro.

El modelo de éste autor funciona bien en estas circunstancias:
- En conflictos entre partes con una relación emocional de baja intensidad.

- Cuando la relación futura entre las partes no tiene demasiada importancia.

- Cuando el objeto del conflicto se puede dividir (por ejemplo dinero).

- Cuando se busque un proceso muy dirigido que casi fuerce a llegar a un acuerdo.

Para Lederach (1984) el conflicto es un fenómeno de creación humana, ubicado de forma natural en las relaciones. Transforma los acontecimientos, las relaciones en las que tiene lugar e, incluso, a sus propios creadores. Es un elemento necesario en la construcción y reconstrucción humana transformadora de la organización de las relaciones sociales.

El mismo autor dice que el conflicto es natural y se experimenta en todas las relaciones y culturas, como pone en juicio a la versión clásica de la resolución de conflictos, articula un paradigma que, analizando el conflicto desde toda su amplitud y profundidad, sustituye el horizonte resolutivo por el transformativo, es decir que la solución del conflicto es la meta, pero no deja de ser más que una meta deseable, pero en ningún caso primordial. La resolución no debe desviarnos de la importancia de la transformación de las propias partes en conflicto cuando se lleva a cabo una gestión positiva.

Esta propuesta se articula desde la gestión positiva del conflicto, que se basa en: Comprensión holística del conflicto: no se juzga por casos específicos, sino que se pretende entender desde toda su amplitud y complejidad.

Negociar desde los intereses y las necesidades de las partes: posibilita construir una nue- 
va realidad en las partes confrontadas para que puedan coexistir, identificando y poniendo sobre la mesa los intereses y necesidades: se abre un abanico de posibles consensos ya que no nos movemos entre posiciones que implican visiones incompatibles.

No es tan importante llegar a un acuerdo si, una vez gestionado el conflicto, se han conseguido preservar las relaciones y vínculos entre las partes.

La Escuela de Harvard, es pionera en materia de medios alternativos de resolución de conflictos, dice que: El conflicto es atemporal, acultural y apersonal, por lo que deberá desaparecer. Se asimila a un problema y, como problema, la solución es factible.

Así tenemos que los conflictos son situaciones en que se manifiesta de manera aguda, la diferencia de opinión de al menos dos personas. Los conflictos son formas de interacción en cuanto implican la presencia de por lo menos dos partes: personas, grupos sociales o incluso Estados.

El conflicto implica, por lo tanto, la interrelación mínimo de dos personas, dos organizaciones o dos grupos sociales cuyos intereses y aspiraciones sean antagónicos, ya sea de modo ocasional o permanente. Parece lo más probable, según la experiencia histórica, que en la medida en que haya interacción social, sea entre personas, grupos o entidades políticas, habrá también ocasiones de disputa: por recursos materiales o simbólicos, más o menos concretos o vagos; por ambiciones contrastantes, por insatisfacción, el conflicto es una constante humana.

Dada la inmensa variedad de fenómenos sociales que pueden clasificarse bajo el término "conflicto", no hay consenso sobre una definición única, general y suficiente, como mencionábamos líneas arriba con los autores. Algunas definiciones sólo explican la esencia de los conflictos interpersonales (dos o más personas, grupo u organizaciones diversas). Otras identifican los rasgos más generales que permiten englobar cualquier tipo de conflicto, sea cual sea en el plano que se produzca. Dichas similitudes permiten que las diversas clases de conflicto se entiendan, en una primera aproximación como expresiones diferentes de un mismo fenómeno social.

\section{3.- El conflicto en la actualidad.}

El conflicto en la actualidad comienza a ser visto como una manifestación más, natural y hasta necesaria, de las relaciones entre personas y entre grupo sociales, entre organismos políticos o Estados. Ya no considera a priori que el conflicto deba conducir necesariamente hacia una ruptura catastrófica de la relación entre las partes que divergen.

Esta nueva forma de ver el conflicto, y por ende de estudiar las relaciones que se establecen entre los individuos $u$ organismos políticos, supone que la interacción produce necesariamente conflicto, porque la rivalidad, la competencia y la diferencia de opiniones, deseos e intereses son inevitables en el orden humano, como de hecho sucede entre todos los seres vivos.

Mientras haya mecanismos y formas que regulen el conflicto, éste puede ser incluso útil, en la medida en que la diferencia que motiva el conflicto permite la evolución y la trasformación de las relaciones entre las partes (Puntes, 2007; Redorta, 2006).

El conflicto así visto ayuda a regular las relaciones sociales; permite el reconocimiento de las diferencias y que éstas no sean percibidas como amenaza, sino como resultado natural de una situación en que hay recursos escasos. 
En sus formas extremas, puede ser en efecto muy destructivo; en sus manifestaciones regulares, contribuye a definir identidades y a racionalizar las estrategias de cooperación y competencia.

El conflicto es una manifestación del orden en el que se produce, de lo cual se derivan dos consecuencias principales: que el orden incluye formas del conflicto, y que el conflicto es una forma ordenada, que sigue pautas.

Los conflictos que se expresan en una sociedad son manifestación del orden, del sistema de relaciones en el que se ha constituido y se desarrolla dicha sociedad; los conflictos regulan el orden, y éste a su vez permite que la regulación de los conflictos cree orden.

El orden en toda sociedad humana, no es otra cosa sino un arreglo normalizado del conflicto; el orden dispone los procedimientos de distribución de recursos. Es decir define qué es objeto de discusión o de competencia o confrontación, qué instrumentos y mecanismos son válidos para decidir, y qué límites tiene el enfrentamiento. De una huelga o un divorcio hasta una competencia electoral, todo sucede dentro del orden.

Todo nuevo orden surge de un orden anterior y por lo tanto está definido y determinado por él; por otra parte encontraban serias dificultades para explicar lo que sucede en situaciones revolucionarias o de convulsión social.

Las formas tan diversas en las que pueden manifestarse los conflictos dependen por entero del carácter de la interacción social que les dio lugar y de la percepción que sobre sí y sobre los demás tienen los individuos que componen el conjunto social.

Sólo el estudio y la disección de las relacio- nes que existen dentro del orden pueden permitir una compresión cabal de los conflictos que en él se originen. No basta con comprender el conflicto en sí, ni el motivo que le dio origen, ni la manera que tiene de manifestarse; es imprescindible conocer las relaciones anteriores, las expectativas de las partes y sus posiciones relativas.

Conocer los antecedentes de las relaciones entre los sujetos para comprender íntegramente el conflicto en el que están sumidos.

En todo conflicto existe un orden, definido por las estrategias de las partes, su posición relativa, la valoración social de los objetos en disputa y los procedimientos para seguirla.

Es conveniente tener en cuenta que, en muchas áreas donde se ha formalizado la interacción humana, el conflicto está regulado de tal modo que no siempre nos damos cuenta siquiera de su existencia. Un ejemplo, fácil de comprender es el del mercado, que regula la competencia entre los seres humanos por los recursos; no elimina el conflicto, sino que lo organiza a través de leyes y normas, de obligado cumplimiento para todos, que excluyen la violencia e incluso se ofrecen como modelo del comportamiento pacíficoy cooperativo. Existe el conflicto pero se regula por medio de las leyes.

Entiende que todo conflicto es resultado de un comportamiento objetivo, pero que no sólo depende de ello, sino también de la manera como las partes involucradas perciben sus intereses.

Ross (1995), lo dice con claridad al señalar que el conflicto "tiene que ver con los fines concretos que los adversarios persiguen y, al mismo tiempo, con las interpretaciones que éstos hacen de lo que está dispuesta". 
El concepto de interés, pues, sirve para entender la estructura del comportamiento, siempre y cuando se tome en cuenta que no se refiere sólo a objetos materiales, calculables.

Así, las personas o los grupos que participan en un conflicto no están exentos de esa variante subjetiva determinada por el entorno y el contexto de cada individuo en la sociedad.

En todo conflicto existe una dimensión subjetiva, determinada por la percepción que las partes tienen del mismo, que influye de manera decisiva en su dinámica y en su posible resolución. Esto no quiere decir que un conflicto sólo aparecerá si las partes así lo deciden, con independencia de que existan o no bases objetivas para sustentarlo.

Los intereses que las partes de un conflicto creen tener y la manera como los perciben suelen estar íntimamente relacionados. Ejemplo: en el reparto de bienes tras un divorcio, quedarse con la casa común puede ser vital para cualquiera de os dos cónyuges si el inmueble marca la diferencia entre "ganar" o "perder" en la negociación.

El análisis del conflicto requiere no sólo atender el asunto que lo origina, sino también profundizar en la relación entre las partesy en el significado que para cada una tiene la disputa. Sólo cuando se lo entiende cabal e íntegramente se puede intervenir con razonable confianza en la posibilidad de resolverlo.

El conflicto en cualquiera de sus formas es uno de los más grandes temas comunes a todas las ciencias sociales y humanas en la actualidad. Existe además en este terreno un ánimo común: ya se trate de conflictos internacionales o de pleitos entre vecinos, familias, empresas, se ve la preocupación compartida por diseñar mecanismos que favorezcan la solución pacífica de controversias.
En su orientación pragmática, casi todas las disciplinas procuran desarrollar técnicas de intervención que favorezcan el diálogo, la comunicación, el acuerdo negociado y el compromiso con una solución satisfactoria para todas las partes. Ningún país puede prescindir de un sistema de justicia eficiente y que vele por el cumplimiento efectivo de la ley.

\section{A manera de reflexión final}

Los conflictos son parte de la vida de forma individual o colectiva, pero va más allá de las personas puesto que engloban a instituciones, países y espacios regionales en los bloques del mundo actual. En tal sentido el análisis que debe realizarse debe partir desde enfoques multidisciplinarios, ya que la presencia del mismo se encuentra en toda organización social.

El conflicto visto desde la perspectiva positiva que no positivista, nos lleva a replantear situaciones a los nuevos sujetos sociales, en tal sentido que nos permite transformar las relaciones sociales y nos ayuda a crecer, pero además a crear mecanismos desde una cultura de diálogo y construcción de espacios de paz. 


\section{Referencias}

Alzate, R. (1998). Análisis y resolución de conflictos. Una perspectiva psicológica. España: Universidad País Vasco.

Arboleda, A., Garcés, L., Murillo, E. \& Pineda, M. (2017). Principios, habilidades y virtudes para el conciliador en Derecho. Pensamiento Americano, 10(18), 189-198. http:// dx.doi.org/10.21803\%2Fpenamer.10.18.394

Baruch, R. A. \& Folger, J. P. (1996). La promesa de la Mediación. Barcelona, España: Editorial Granica,

Cobb, S. (1995). La Neutralidad como práctica discursiva. La construcción y transformación de narrativas en la mediación en comunidades. Material bibliográfico del curso "Negociación y resolución de conflictos", Universidad de California, Santa Bárbara.

Constitución Política de los Estados Unidos Mexicanos. (2011). DOF 10 de junio de 2011.

Cornelio, E. (2017). Mediación: mecanismo para la solución de conflictos laborales en México. Horizontalidad de la justicia, México: Editorial Porrúa,

De Bono, Edwar. (2012). Seis Sombreros para pensar. Ediciones Granica, España.

Gottheil, J. \& Schiffrin, A. (1996). Mediación. Una transformación en la cultura, Buenos Aires, Argentina: Paidós,

Hernández, H. (2007). El convenio de mediación, Comisión de Derechos Humanos del Estado de México: Centro de Estudios.

Hoyo, I. (2006). La idoneidad de los métodos alternativos de solución de conflictos. Seccion: Contribuciones Métodos alternativos de solución de conflictos: perspectiva multidisciplinar Lex. p. 356.

Katz, N. (1993). Conflict resolution: building bridges, Syracuse University, USA.

Lederach, J. (1984). Educar para la paz, Barcelona, España: Editorial Fontamara.

Martínez, B. (1999). Mediación y resolución de conflictos, una guía introductoria. México: Editorial Paidós,

Moore, C. (1996). El proceso de Mediación. Buenos Aires,
Argentina: Granica.

Navarro, L. \& Romero, M. (2016). Los conceptos de poder y violencia en Hannah Arendt: un análisis desde la comunicación. Pensamiento Americano, 9(17), 54-66.

Puntes, S. (2007). La mediación comunitaria: ciudadanos, derechos y conflictos. Bogotá, Colombia: Cámara de Comercio de Bogotá;

Real Academia Española. (2001). Diccionario de la lengua española (22a ed.). Recuperado de: http://mwwrae.es/ rae.html.

Redorta, J. (2004). Como analizar Conflictos. Barcelona, España: Ediciones Paidós.

Redorta, J. (2006). Emoción y conflicto. Barcelona, España: Ediciones Paidós.

Rodríguez Casallas, D. F., \& Arango Morales, X. (2017). Despolitización y ética pública. La crisis del pensarse en colectivo en la modernidad líquida. Justicia, (31), 6586. https://dx.doi.org/10.17081/just.22.31.2599

Ross, M. (1995). La cultura del conflicto: las diferencias interculturales en la práctica de la violencia, España: Paidós.

Suáres, M. (1996). Mediación, conducción de disputas, comunicación y técnicas. Quilmes, Argentina: Paidós.

Ury, W. (1996). Supere el no. Colombia: Grupo Editorial Norma.

Vélez Vélez, J. (2017). La ciudadanía como condición humana. Revista Pensamiento Americano, 10(18). 133141.

Vinyamata, E. (2005). Conflictología. Barcelona, España: Editorial Ariel.

Wright, R. (2012). Psicología Social para Todos: tierra y escritura del hacer, sentir y pensar - Año 4 Nro. 38 de marzo.

2018, Vol. 12(23) 169- 182 @The Author(s) 2018 Reprints and permission: www.americana.edu.co

https://www.coruniamericana.edu.co/publicaciones/ojs/index.php/pensamientoamericano/index 